\title{
THE FRACTIONAL MIXED FRACTIONAL BROWNIAN MOTION AND FRACTIONAL BROWNIAN SHEET
}

\author{
Charles El-Nouty ${ }^{1}$
}

\begin{abstract}
We introduce the fractional mixed fractional Brownian motion and fractional Brownian sheet, and investigate the small ball behavior of its sup-norm statistic. Then, we state general conditions and characterize the sufficiency part of the lower classes of some statistics of the above process by an integral test. Finally, when we consider the sup-norm statistic, the necessity part is given by a second integral test.
\end{abstract}

Mathematics Subject Classification. 60F15, 60G15.

Received September 29, 2005. Revised May 2, 2006.

\section{INTRODUCTION AND MAIN RESULTS}

Let $\left\{B_{H_{1}}(s), s \geq 0\right\}$ be a fractional Brownian motion (FBM) with index $0<H_{1}<1$, i.e. a centered Gaussian process with stationary increments satisfying $B_{H_{1}}(0)=0$, with probability 1 , and $\mathbb{E}\left(B_{H_{1}}(s)\right)^{2}=s^{2 H_{1}}, s \geq 0$. Denote by $\sigma_{H_{1}}$ the covariance function of $B_{H_{1}}$. Moreover, recall that $B_{H_{1}}$ can be represented as a random integral, i.e.

$$
B_{H_{1}}(s)=\int_{\mathbb{R}} g_{H_{1}}(s, u) \tilde{W}(\mathrm{~d} u)
$$

where $\tilde{W}(u), u \in \mathbb{R}$, is a Wiener process,

$$
g_{H_{1}}(s, u)=k_{2 H_{1}}^{-1}\left(\max (s-u, 0)^{H_{1}-1 / 2}-\max (-u, 0)^{H_{1}-1 / 2}\right),
$$

and $k_{2 H_{1}}$ is a normalizing constant. We refer to Li and Shao [18] for further information on this field.

A natural extension of $B_{H_{1}}$ in 2-dimensional space is given by

$$
B_{H_{2}, H_{3}}\left(s_{2}, s_{3}\right)=\int_{-\infty}^{s_{2}} \int_{-\infty}^{s_{3}} g_{H_{2}}\left(s_{2}, u_{2}\right) g_{H_{3}}\left(s_{3}, u_{3}\right) W\left(\mathrm{~d}\left(u_{2}, u_{3}\right)\right)
$$

where $W\left(u_{2}, u_{3}\right), u_{2} \in \mathbb{R}, u_{3} \in \mathbb{R}$, is a standard Brownian sheet and $0<H_{2}, H_{3}<1$. Its covariance function $\sigma_{H_{2}, H_{3}}$ is given by

$$
\sigma_{H_{2}, H_{3}}\left(\left(s_{2}, s_{3}\right),\left(s_{2}^{\prime}, s_{3}^{\prime}\right)\right)=\sigma_{H_{2}}\left(s_{2}, s_{2}^{\prime}\right) \times \sigma_{H_{3}}\left(s_{3}, s_{3}^{\prime}\right)
$$

Keywords and phrases. Fractional Brownian motion, fractional Brownian sheet, lower classes, small ball probabilities.

1 U.F.R. de mathématiques, Université Paris VI, 175 rue du Chevaleret, 75013 Paris, France; elnouty@ccr.jussieu.fr

(C) EDP Sciences, SMAI 2007 
$B_{H_{2}, H_{3}}$ is named the fractional Brownian sheet (FBS). There is a huge literature on this process. We refer to Ayache et al. [1], Ayache and Xiao [2], Belinsky and Linde [3], Kühn and Linde [14], Mason and Shi[20] and Xiao and Zhang [25] for further information on the FBS.

The study of the FBM and its extension was motivated by natural time series in economics, fluctuations in solids, hydrology and more recently by new problems in mathematical finance, telecommunication networks and the environment.

In the sequel, we assume that $B_{H_{1}}$ and $B_{H_{2}, H_{3}}$ are independent. Let $\lambda_{1}$ and $\lambda_{2}$ be two real numbers such that $\lambda_{1} \lambda_{2} \neq 0$. In the spirit of Cheridito [5] and El-Nouty [9], we introduce the fractional mixed fractional Brownian motion and fractional Brownian sheet (FMFBMFBS) defined as follows

$$
X\left(w_{1}, w_{2}, w_{3}, s\right)=\lambda_{1} s^{H_{2}+H_{3}} B_{H_{1}}\left(w_{1}\right)+\lambda_{2} s^{H_{1}} B_{H_{2}, H_{3}}\left(w_{2}, w_{3}\right),
$$

and consider the sup-norm statistic

$$
Y(t)=\sup _{0 \leq s \leq t} \sup _{0 \leq w_{1}, w_{2}, w_{3} \leq s}\left|X\left(w_{1}, w_{2}, w_{3}, s\right)\right|, \quad t \geq 0 .
$$

Note first that, by the scaling property, we have for any $\epsilon>0$

$$
\begin{aligned}
\mathbb{P}\left(Y(t) \leq \epsilon t^{H_{1}+H_{2}+H_{3}}\right) & =\mathbb{P}\left(\sup _{0 \leq s \leq 1} \sup _{0 \leq w_{1}, w_{2}, w_{3} \leq s}\left|X\left(w_{1}, w_{2}, w_{3}, s\right)\right| \leq \epsilon\right) \\
& =\mathbb{P}(Y(1) \leq \epsilon):=\phi(\epsilon),
\end{aligned}
$$

where $\phi$ is named the small ball function and $\gamma:=H_{1}+H_{2}+H_{3}$ the scaling factor.

The motivation supporting this paper is threefold:

- The first goal of the FMFBMFBS deals with the potential applications to the above mentioned fields. Since the FMFBMFBS can be analyzed based on the large bodies of knowledge on FBM and FBS, it can be used in the same fields. This may look like a tautology, but this remark applies to fractional mixed fractional Gaussian processes (i.e. a suitable combination of some appropriate fractional Gaussian processes). For example, to modelize the discounted stock price, the fractional version of the Samuelson model [23] was studied by Cutland et al. [6]. But, since it had also some deficiencies, Cheridito [5] introduced some mixed fractional Gaussian processes. The FMFBMFBS could be used to modelize the diffusion of atmospheric pollutants, either accidental (nuclear, chemical) or not (air pollution). To validate this model, we could compare the theoretical results to those obtained by Gassmann and Bürki [12] and Gassmann et al. [13].

- A second application deals with the small ball probability problem of the sum of two joint centered Gaussian random vectors $X$ and $Y$ in a separable Banach space $E$ with norm $\|$.$\| . This problem was$ investigated in $\mathrm{Li}$ [16], when $X$ and $Y$ are not necessarily independent and have a standard small ball factor $\left(c f\right.$. El-Nouty [7,11]). Here we assume $B_{H_{1}}$ and $B_{H_{2}, H_{3}}$ are independent but $B_{H_{2}, H_{3}}$ can have a log-type small ball factor (cf. El-Nouty [11]). Thus, the study of the small ball behavior of the FMFBMFBS gives a first answer of the small ball probability problem of the sum of two centered independent Gaussian random vectors, having a log-type small ball factor.

- Last but not least, this paper extends El-Nouty's results [7-11] and consequently answers some new questions. Recall first two definitions of the Lévy classes, stated in Révész [22]. Let $\{Z(t), t \geq 0\}$ be a stochastic process defined on the basic probability space $(\Omega, \mathcal{A})$. 
Definition 1.1. The function $f(t), t \geq 0$, belongs to the lower-lower class of the process $Z,(f \in L L C(Z))$, if for almost all $\omega \in \Omega$ there exists $t_{0}=t_{0}(\omega)$ such that $Z(t) \geq f(t)$ for every $t>t_{0}$.

Definition 1.2. The function $f(t), t \geq 0$, belongs to the lower-upper class of the process $Z,(f \in L U C(Z))$, if for almost all $\omega \in \Omega$ there exists a sequence $0<t_{1}=t_{1}(\omega)<t_{2}=t_{2}(\omega)<\ldots$ with $t_{n} \rightarrow+\infty$, as $n \rightarrow+\infty$, such that $Z\left(t_{n}\right) \leq f\left(t_{n}\right), n \in \mathbb{N}^{*}$.

In the spirit of Talagrand [24] and El-Nouty [7-11], the main aim of this paper is to characterize the lower classes of $Y$ for any $0<H_{1}, H_{2}, H_{3}<1$. El-Nouty [7] characterized the lower classes of a large class of statistics of the FBM. The role of a log-type small ball factor was studied in El-Nouty [11] by considering the FBS. Here the FMFBMFBS enables us to compare the influence of the FBM and the FBS, and consequently to measure the weight of a standard type small ball factor versus a log-type one.

Set $\alpha=\min \left(H_{1}, H_{2}, H_{3}\right)$, which is in $] 0,1[$. We introduce the number $\beta$ taking its values in $\{0,1+1 / \alpha\}$. When $H_{2} \neq H_{3}$ or $H_{1}<H_{2}=H_{3}, \beta=0$, whereas, when $H_{2}=H_{3} \leq H_{1}, \beta=1+1 / \alpha$. The small ball behavior of the FMFBMFBS is given in the first result.

Theorem 1.1. There is a constant $K_{0}, 0<K_{0} \leq 1$, depending on $H_{1}, H_{2}, H_{3}, \lambda_{1}$ and $\lambda_{2}$ only, such that we have for $0<\epsilon<1$

$$
\exp \left(-\frac{(\log (1 / \epsilon))^{\beta}}{K_{0} \epsilon^{1 / \alpha}}\right) \leq \phi(\epsilon) \leq \exp \left(-\frac{K_{0}(\log (1 / \epsilon))^{\beta}}{\epsilon^{1 / \alpha}}\right) .
$$

Note first that the minimum $\alpha$ plays a key role. This is not really surprising. Indeed this phenomenon was already observed in El-Nouty [9]. We can also remark that, when $\beta=1+1 / \alpha$, we have a log-type small ball factor. This is a consequence of the small ball behavior of the FBS which was studied by Belinsly and Linde [3] and Mason and Shi [20]. Recall also that the existence of small ball constants for the FBM was showed by $\mathrm{Li}$ and Linde [17]. Hence, when $H_{1} \leq \min \left(H_{2}, H_{3}\right)$, Theorem 1.1 (and consequently the sharpness of Ths. 1.2 and 1.3) can be improved by establishing the existence of the small ball constant for the FMFBMFBS.

It appears that the sufficiency part of the lower classes of $Y$ can be stated in a general framework. Roughly speaking, we follow the same lines as those of El-Nouty [7,11].

Let $\left\{Y_{0}(t), t \geq 0\right\}$ be a real-valued statistic of $B_{H_{1}}$ and $B_{H_{2}, H_{3}}$, such that $Y_{0}(t)$ is a nondecreasing function of $t \geq 0$.

The following notation is needed. If $\mathbb{K}$ is a Hausdorff compact space, we denote by $C(\mathbb{K})$ the space of all continuous functions from $\mathbb{K}$ to $\mathbb{R}$ equipped with the classical sup-norm. Let $\boldsymbol{X}=C([0,1]) \times C\left([0,1]^{2}\right)$ be the product space equipped with the product topology. Denote by $\mathrm{L}\left(B_{H_{1}}, B_{H_{2}, H_{3}}\right)$ the Gaussian measure associated to $B_{H_{1}}$ and $B_{H_{2}, H_{3}}$ and defined on $\mathbb{B}$, the Borel $\sigma$-field of $\boldsymbol{X}$.

We assume that $Y_{0}$ satisfies the three following conditions : (C1) The scaling condition. There exists $\gamma_{0}>0$ such that

$$
\mathbb{P}\left(Y_{0}(t) \leq \epsilon t^{\gamma_{0}}\right)=\mathbb{P}\left(Y_{0}(1) \leq \epsilon\right):=\phi(\epsilon)
$$

(C2) The convexity condition. There exists a convex and $\mathbb{B}$-measurable function $g:\left(\boldsymbol{X}, \mathrm{L}\left(B_{H_{1}}, B_{H_{2}, H_{3}}\right)\right) \rightarrow \mathbb{R}$ such that for any $t \geq 0, Y_{0}(t)=g\left(B_{H_{1}}\left(s_{1} t\right), B_{H_{2}, H_{3}}\left(s_{2} t, s_{3} t\right) ; 0 \leq s_{1}, s_{2}, s_{3} \leq 1\right)$, and $Y_{0}(t)<+\infty$, with probability 1 . 
(C3) The log-type small ball condition. There exist $\left.\left.\alpha_{0} \in\right] 0, \gamma_{0}\right], \beta_{0} \in \mathbb{R}$ and a constant $K, 0<K \leq 1$, depending on $\gamma_{0}, \alpha_{0}$ and $\beta_{0}$ only, such that we have for $0<\epsilon<1$

$$
\exp \left(-\frac{(\log (1 / \epsilon))^{\beta_{0}}}{K \epsilon^{1 / \alpha_{0}}}\right) \leq \phi(\epsilon) \leq \exp \left(-\frac{K(\log (1 / \epsilon))^{\beta_{0}}}{\epsilon^{1 / \alpha_{0}}}\right) .
$$

Note that these conditions generalize those of El-Nouty [7,11]. The small ball function still plays a key role. The convexity of the function $\psi$ defined by $\psi(\epsilon)=-\log \phi(\epsilon), 0<\epsilon<1$, is ensured by the condition $(C 2)$ (see Borell [4], p. 243, Ledoux and Talagrand [15] and Lifshits [19], pp. 108-137).

Our second result is given in the following theorem.

Theorem 1.2. Let $f(t)$ be a positive nondecreasing function of $t \geq 0$. Assume that there exists $m>0$ such that $\frac{f(t)}{t^{\gamma_{0}-\alpha_{0}}}\left(\log \frac{t^{\gamma_{0}}}{f(t)}\right)^{-\beta_{0} \alpha_{0}} \geq m$.

If

$$
\frac{f(t)}{t^{\gamma_{0}}} \text { is bounded and } \int_{0}^{+\infty} f(t)^{-1 / \alpha_{0}} t^{\left(\gamma_{0} / \alpha_{0}\right)-1}\left(\log \frac{t^{\gamma_{0}}}{f(t)}\right)^{\beta_{0}} \phi\left(\frac{f(t)}{t^{\gamma_{0}}}\right) \mathrm{d} t<+\infty .
$$

then we have

$$
f \in \operatorname{LLC}\left(Y_{0}\right)
$$

The sup-norm statistic $Y$ clearly satisfies the three above conditions with $\gamma_{0}=\gamma=H_{1}+H_{2}+H_{3}, \alpha_{0}=\alpha=$ $\min \left(H_{1}, H_{2}, H_{3}\right), \beta_{0}=\beta \in\{0,1+1 / \alpha\}$ and $K=K_{0}$. Now, we characterize the necessity part of the lower classes of the FMFBMFBS. Our main result is stated in the following theorem.

Theorem 1.3. Let $f(t)$ be a positive nondecreasing function of $t \geq 0$ such that $\frac{f(t)}{t^{\gamma}}$ is a nonincreasing function of $t>0$.

If

then we have

$$
f \in L L C(Y)
$$

$$
\lim _{t \rightarrow+\infty} \frac{f(t)}{t^{\gamma}}=0 \text { and } \int_{0}^{+\infty} f(t)^{-1 / \gamma} \phi\left(\frac{f(t)}{t^{\gamma}}\right) \mathrm{d} t<+\infty .
$$

First, we can notice that Theorem 1.2 depends on $\gamma_{0}, \alpha_{0}$ and $\beta_{0}$. If $\beta_{0}=0$, Theorem 1.2 looks like Theorem 1 of El-Nouty [7], p. 365, or else like Theorem 1.1 of El-Nouty [11], p. 321. As expected, Theorem 1.3 has the same form as the theorems obtained by Talagrand [24] and El-Nouty [7-11]. The methodology of Talagrand [24] can lead to two integral tests in the study of the lower classes of $Y$. But Theorems 1.2 and 1.3 are sharp. Indeed, set, if $\beta=0$,

or else (i.e. $\beta=1+(1 / \alpha))$

$$
f(t)=\frac{\lambda t^{\gamma}}{(\log \log t)^{\alpha}}, t \geq 3, \lambda>0 .
$$

$$
f(t)=t^{\gamma} \frac{(\lambda \log \log \log t)^{1+\alpha}}{(\log \log t)^{\alpha}}, t \geq 16, \lambda>0 .
$$

If $\lambda$ is small enough, then Theorem 1.2 yields $f \in L L C(Y)$, and if $\lambda$ is large enough, then $f \in L U C(Y)$ by applying Theorem 1.3. 
In Section 2, we prove Theorem 1.1. The proof of Theorem 1.3 is postponed to Sections 3 and 4 and will be given in details. In Section 4 we establish some key small ball estimates. Note also that these estimates can be of independent interest. The proofs which are modifications of those of El-Nouty [7,11] will be consequently omitted, in particular the proof of Theorem 1.2.

In the sequel, there is no loss of generality to assume that $H_{2} \leq H_{3}$.

\section{Proof of Theorem 1.1}

The proof will be split into two parts: the lower bound and the upper one.

Part I. The lower bound. We have

$\phi(\epsilon) \geq \mathbb{P}\left(\left\{\sup _{0 \leq s \leq 1} \sup _{0 \leq w_{1} \leq s}\left|\lambda_{1} s^{H_{2}+H_{3}} B_{H_{1}}\left(w_{1}\right)\right| \leq \frac{\epsilon}{2}\right\}\right.$

Hence we get by independence and monotonicity

$$
\left.\bigcap\left\{\sup _{0 \leq s \leq 1} \sup _{0 \leq w_{2}, w_{3} \leq s}\left|\lambda_{2} s^{H_{1}} B_{H_{2}, H_{3}}\left(w_{2}, w_{3}\right)\right| \leq \frac{\epsilon}{2}\right\}\right) .
$$

$$
\phi(\epsilon) \geq \mathbb{P}\left(\sup _{0 \leq w_{1} \leq 1}\left|B_{H_{1}}\left(w_{1}\right)\right| \leq \frac{\epsilon}{2\left|\lambda_{1}\right|}\right) \times \mathbb{P}\left(\sup _{0 \leq w_{2}, w_{3} \leq 1}\left|B_{H_{2}, H_{3}}\left(w_{2}, w_{3}\right)\right| \leq \frac{\epsilon}{2\left|\lambda_{2}\right|}\right) .
$$

Combining Belinsky and Linde [3], Mason and Shi [20] Monrad and Rootzen [21] and Talagrand [24] with (2.1), we obtain that:

if $H_{2}=H_{3} \leq H_{1}$, then $\phi(\epsilon) \geq \exp \left(-\frac{(\log (1 / \epsilon))^{1+(1 / \alpha)}}{C_{1} \epsilon^{1 / \alpha}}\right)$

$$
\text { or else } \phi(\epsilon) \geq \exp \left(-\frac{1}{C_{2} \epsilon^{1 / \alpha}}\right)
$$

where $C_{1}$ and $C_{2}$ are strictly positive constants.

The proof of the lower part of Theorem 1.1 is complete.

Part II. The upper bound. By choosing $s=1$, we get

$$
\phi(\epsilon) \leq \mathbb{P}\left(\sup _{0 \leq w_{1}, w_{2}, w_{3} \leq 1}\left|\lambda_{1} B_{H_{1}}\left(w_{1}\right)+\lambda_{2} B_{H_{2}, H_{3}}\left(w_{2}, w_{3}\right)\right| \leq \epsilon\right) .
$$

Since $\left\{\left(w_{1}, 0,0\right): 0 \leq w_{1} \leq 1\right\} \subset[0,1]^{3}$ and $\left\{\left(0, w_{2}, w_{3}\right): 0 \leq w_{2}, w_{3} \leq 1\right\} \subset[0,1]^{3}$, the following inequality holds

$$
\phi(\epsilon) \leq \inf \left(\mathbb{P}\left(\sup _{0 \leq w_{1} \leq 1}\left|\lambda_{1} B_{H_{1}}\left(w_{1}\right)\right| \leq \epsilon\right), \mathbb{P}\left(\sup _{0 \leq w_{2}, w_{3} \leq 1}\left|\lambda_{2} B_{H_{2}, H_{3}}\left(w_{2}, w_{3}\right)\right| \leq \epsilon\right)\right) .
$$

By considering the four cases $H_{1} \leq H_{2}<H_{3}, H_{2}<H_{3}$ and $H_{2} \leq H_{1}, H_{1}<H_{2}=H_{3}$, and $H_{2}=H_{3} \leq H_{1}$, we use (2.2) and the results in Belinsky and Linde [3], Mason and Shi [20], Monrad and Rootzen [21] and Talagrand [24]. We emphasize the fact that we obtain a log-type small ball factor if and only if $H_{2}=H_{3} \leq H_{1}$.

The proof of Theorem 1.1 is now complete. 


\section{PROOF OF THEOREM 1.3: PART I}

Set $a_{t}=\frac{f(t)}{t^{\gamma}}$ and $b_{t}=\phi\left(a_{t}\right)$.

Suppose here that, with probability $1, f(t) \leq Y(t)$ for all $t$ large enough. We want to prove that $\lim _{t \rightarrow+\infty} a_{t}=0$ and $\int_{0}^{\infty} a_{t}^{-1 / \gamma} b_{t} \frac{\mathrm{d} t}{t}<+\infty$.

In the sequel, there is no loss of generality in assuming that $f$ is a continuous function of $t \geq 0$. Indeed the set of points at which $f$ is discontinuous is at most countable and therefore has measure zero.

Lemma 3.1. We have

$$
\lim _{t \rightarrow+\infty} a_{t}=0
$$

Proof. Let $\lim _{t \rightarrow \infty} a_{t}=c \geq 0$. If $c>0$, then $\lim _{t \rightarrow \infty} b_{t}=\phi(c)>0$, which is impossible. (3.1) is proved.

To prove Theorem 1.3, we will show that $f \in L U C(Y)$ when $\int_{0}^{\infty} a_{t}^{-1 / \gamma} b_{t} \frac{\mathrm{d} t}{t}=+\infty$ and $\lim _{t \rightarrow+\infty} a_{t}=0$.

First recall the following corollary (see Talagrand [24], p. 198).

Corollary A. Assume that $J \subset \mathbb{N}$ and that, for some numbers $K$ and $\epsilon$, we have for the family of sets $\left(A_{i}\right)_{i \in J}$ in a basic probability space

$$
\forall i \in J \quad \sum_{j>i} \mathbb{P}\left(A_{i} \cap A_{j}\right) \leq \mathbb{P}\left(A_{i}\right)\left(K+(1+\epsilon) \sum_{j>i} \mathbb{P}\left(A_{j}\right)\right) .
$$

Then, if

$$
\sum_{i \in J} \mathbb{P}\left(A_{i}\right) \geq \frac{1+2 K}{\epsilon}
$$

we have

$$
\mathbb{P}\left(\bigcup_{i \in J} A_{i}\right) \geq \frac{1}{1+2 \epsilon}
$$

Our aim is to construct a suitable set $J$ which satisfies hypothesis (3.2) and (3.3).

Lemma 3.2. When $\int_{0}^{\infty} a_{t}^{-1 / \gamma} b_{t} \frac{\mathrm{d} t}{t}=+\infty$ and $\lim _{t \rightarrow+\infty} a_{t}=0$, we can find a sequence $\left\{t_{n}, n \geq 1\right\}$ with the two following properties

$$
t_{n+1} \geq t_{n}\left(1+a_{t_{n}}^{1 / \gamma}\right)
$$

and

$$
\sum_{n=1}^{\infty} b_{t_{n}}=+\infty
$$

Proof. For the construction of $\left\{t_{n}, n \geq 1\right\}$, we proceed by induction over $n$. Set $t_{1}=1$. Having constructed $t_{n}$, we define

$$
s_{n}=t_{n}\left(1+a_{t_{n}}^{1 / \gamma}\right)
$$

We set $t_{n+1}=s_{n}\left(1+a_{s_{n}}^{1 / \alpha}\right)$. 
(3.5) is obviously proved.

To prove (3.6), it is enough to show that, for $n$ sufficiently large, we have

$$
I_{n}=\int_{t_{n}}^{t_{n+1}} a_{t}^{-1 / \gamma} b_{t} \frac{\mathrm{d} t}{t} \leq K b_{t_{n}}
$$

where $K$ is a constant.

Set $I_{n 1}=\int_{t_{n}}^{s_{n}} a_{t}^{-1 / \gamma} b_{t} \frac{\mathrm{d} t}{t}$ and $I_{n 2}=\int_{s_{n}}^{t_{n+1}} a_{t}^{-1 / \gamma} b_{t} \frac{\mathrm{d} t}{t}$. Consider first $I_{n 1}$. We obtain

$$
I_{n 1} \leq\left(s_{n}-t_{n}\right) f\left(t_{n}\right)^{-1 / \gamma} \phi\left(a_{t_{n}}\right)=b_{t_{n}}
$$

Consider now $I_{n 2}$. Since $t_{n+1} \leq 2 s_{n}, f$ is nondecreasing and $a_{t}^{-1 / \gamma} \leq a_{t}^{-1 / \alpha}$, we have

$$
I_{n 2} \leq \int_{s_{n}}^{t_{n+1}} a_{t}^{-1 / \alpha} b_{t} \frac{\mathrm{d} t}{t} \leq\left(t_{n+1}-s_{n}\right) f\left(s_{n}\right)^{-1 / \alpha} t_{n+1}^{(\gamma / \alpha)-1} \phi\left(a_{s_{n}}\right) \leq 2^{(\gamma / \alpha)-1} b_{t_{n}}
$$

Hence, we get $I_{n}=I_{n 1}+I_{n 2} \leq\left(1+2^{(\gamma / \alpha)-1}\right) b_{t_{n}}$.

The sequence $\left\{t_{n}, n \geq 1\right\}$ we have constructed is not yet appropriate. We need a further construction (the reason for which will become apparent only later).

We need the following definition and notation.

Definition 3.1. Consider the interval $A_{k}=\left[2^{k}, 2^{k+1}\left[, k \in \mathbb{I}\right.\right.$. If $a_{t_{i}}^{-1 / \gamma} \in A_{k}, i \in \mathbb{N}^{*}$, then we note $u(i)=k$.

\section{Notation.}

1. $I_{k}=\left\{i \in \mathbb{N}^{*}, u(i)=k \in \mathbb{N}\right\}$ which is finite by Lemma 3.1;

2. $N_{k}=\exp \left(K_{0}(\gamma \log 2)^{\beta} k^{\beta} 2^{\gamma(k-1) / \alpha}\right)$, where $K_{0}$ has the same value as in Theorem 1.1;

3. $\left.\left.F_{m, k}=\left\{i \in \mathbb{N}^{*}, i \in I_{k}, m<i, \operatorname{card}\left(I_{k} \cap\right] m, i\right]\right) \leq N_{k}\right\}, m \in \mathbb{N}^{*}, k \in \mathbb{N}$;

4. $k_{0}=\inf \left\{n \in \mathbb{N}, 2^{\gamma n / \alpha} \geq \frac{2^{\gamma / \alpha}}{K_{0}\left(2^{\gamma / \alpha}-1\right)(\gamma \log 2)^{\beta}}+\frac{2^{2 \gamma / \alpha}}{K_{0}^{2}\left(2^{\gamma / \alpha}-1\right)}\right\},\left(k_{0}\right.$ depends on $K_{0}, \gamma$ and $\alpha$ only ;

5. $V_{m}=\bigcup_{k \in \mathbb{N}} F_{m, k}$, where $m$ is fixed, $u(m)=k_{1}$ and $k \geq k_{1}+k_{0}$;

6. $W=\bigcup_{m \geq 1} V_{m}$

Now we can define our set $J$ as follows

$$
J=\mathbb{N}-W
$$

Lemma 3.3. We have $\sum_{n \in J} b_{t_{n}}=+\infty$. 
Given $n \in J, m \in J, n<m$, such that $\operatorname{card}\left(I_{u(n)} \cap[n, m]\right)>\exp \left(K_{0} 2^{u(n)-1}\right)$, we have

$$
\frac{t_{m}}{t_{n}} \geq \exp \left(\exp \left(\frac{K_{0}}{4} 2^{\min (u(n), u(m))}\right)\right)
$$

Proof. We show first

$$
\sum_{i \in V_{m}} b_{t_{i}} \leq \frac{3}{4} b_{t_{m}}
$$

We have for any $i \in I_{k}$

$$
\frac{1}{2^{(k+1) \gamma}} \leq a_{t_{i}} \leq \frac{1}{2^{k \gamma}}
$$

and consequently by Theorem 1.1

$$
\exp \left(-\frac{(\gamma \log 2)^{\beta}(k+1)^{\beta} 2^{\gamma(k+1) / \alpha}}{K_{0}}\right) \leq b_{t_{i}} \leq \exp \left(-K_{0}(\gamma \log 2)^{\beta} k^{\beta} 2^{\gamma k / \alpha}\right)
$$

Hence, we have

$$
\sum_{i \in F_{m, k}} b_{t_{i}} \leq N_{k} \exp \left(-K_{0}(\gamma \log 2)^{\beta} k^{\beta} 2^{\gamma k / \alpha}\right) \leq \exp \left(-\left(2^{\gamma / \alpha}-1\right) K_{0}(\gamma \log 2)^{\beta} k^{\beta} 2^{\gamma(k-1) / \alpha}\right) .
$$

Then, we obtain

$$
\begin{gathered}
\sum_{i \in V_{m}} b_{t_{i}} \leq b_{t_{m}} \sum_{k=k_{0}+k_{1}}^{+\infty} \frac{1}{b_{t_{m}}} \exp \left(-\left(2^{\gamma / \alpha}-1\right) K_{0}(\gamma \log 2)^{\beta} k^{\beta} 2^{\gamma(k-1) / \alpha}\right) \\
\leq b_{t_{m}} \sum_{k=k_{0}+k_{1}}^{+\infty} \exp \left((\gamma \log 2)^{\beta}\left(k_{1}+1\right)^{\beta}\left(\frac{2^{\gamma\left(k_{1}+1\right) / \alpha}}{K_{0}}-K_{0}\left(2^{\gamma / \alpha}-1\right) 2^{\gamma(k-1) / \alpha}\right)\right)
\end{gathered}
$$

Setting $l=k-\left(k_{0}+k_{1}\right)$ and recalling the definition of $k_{0}$, we get

$$
\begin{aligned}
& (\gamma \log 2)^{\beta}\left(\frac{2^{\gamma\left(k_{1}+1\right) / \alpha}}{K_{0}}-K_{0}\left(2^{\gamma / \alpha}-1\right) 2^{\gamma(k-1) / \alpha}\right) \\
& \leq 2^{\gamma k_{1} / \alpha}\left(\frac{2^{\gamma / \alpha}(\gamma \log 2)^{\beta}}{K_{0}}-2^{\gamma l / \alpha}-\frac{2^{\gamma(l+1) / \alpha}(\gamma \log 2)^{\beta}}{K_{0}}\right) \leq-2^{\gamma\left(l+k_{1}\right) / \alpha} \leq-2^{l+k_{1}} .
\end{aligned}
$$

Combining (3.10) with (3.11), we get

$$
\sum_{i \in V_{m}} b_{t_{i}} \leq b_{t_{m}} \sum_{l=0}^{+\infty} \exp \left(-2^{l}\right) \leq b_{t_{m}} \sum_{l=0}^{+\infty} \frac{3}{42^{l+1}}=\frac{3}{4} b_{t_{m}}
$$

Hence (3.9) is proved.

$$
\text { Let } p \in \mathbb{N}, J_{p}=J \cap\left(\bigcup_{0 \leq k_{1} \leq p} I_{k_{1}}\right) \text { and } W_{p}=W \cap\left(\bigcup_{0 \leq k_{1} \leq p} I_{k_{1}}\right)=\left(\bigcup_{0 \leq k_{1} \leq p} I_{k_{1}}\right)-J_{p} .
$$


The definition of $W_{p}$ and (3.9) yield

$$
\sum_{i \in W_{p}} b_{t_{i}} \leq \sum_{k_{1}=0}^{p} \sum_{m \in I_{k_{1}}} \sum_{i \in V_{m}} b_{t_{i}} \leq \frac{3}{4} \sum_{k_{1}=0}^{p} \sum_{m \in I_{k_{1}}} b_{t_{m}}=\frac{3}{4} \sum_{m \in\left(\cup_{0 \leq k_{1} \leq p} I_{k_{1}}\right)} b_{t_{m}}
$$

Since $\left(\bigcup_{0 \leq k_{1} \leq p} I_{k_{1}}\right)$ is finite, we have

$$
\sum_{i \in J_{p}} b_{t_{i}} \geq \frac{1}{4} \sum_{m \in\left(\bigcup_{0 \leq k_{1} \leq p} I_{k_{1}}\right)} b_{t_{m}}
$$

Let $p \rightarrow+\infty$. Then, we have $\frac{1}{4} \sum_{m \in\left(\bigcup_{0 \leq k_{1} \leq p} I_{k_{1}}\right)} b_{t_{m}} \rightarrow+\infty$ by Lemma 3.2 , and $J_{p} \rightarrow J$.

Hence (3.7) is established.

To prove (3.8), set $k=u(n), k_{1}=u(m)$ and $G=I_{k} \cap[n, m]=\left\{i_{1}, i_{2}, \ldots, i_{z}\right\}$ where $n \leq i_{1}<i_{2}<\ldots<i_{z} \leq m$. We have

$$
\frac{t_{m}}{t_{n}}=\frac{t_{m}}{t_{i_{z}}} \frac{t_{i_{z}}}{t_{i_{z-1}}} \ldots \frac{t_{i_{1}}}{t_{n}}
$$

Note that, when $i \in I_{k}$, we have $t_{i+1} \geq t_{i}\left(1+a_{t_{i}}^{1 / \gamma}\right) \geq t_{i}\left(1+2^{-k-1}\right)$. Moreover, since $\operatorname{card}(G)>\exp \left(K_{0} 2^{k-1}\right)$ by hypothesis, (3.12) implies

$$
\frac{t_{m}}{t_{n}} \geq \exp \left(\exp \left(K_{0} 2^{k-1}\right) \log \left(1+2^{-k-1}\right)\right) \geq \exp \left(\exp \left(\frac{K_{0}}{4} 2^{k}\right)\right)
$$

when $n$, hence $k$, are large enough.

Thus, whenever $k_{1} \leq k+k_{0}$, (3.13) implies (3.8).

Next assume $k_{1}>k+k_{0}$. Since $m \in J, m \notin V_{n}$, and consequently $n \notin F_{n, k_{1}}$. Thus $\operatorname{card}\left(I_{k_{1}} \cap[n, m]\right)>N_{k_{1}}$. When $n$, hence $m$ and $k_{1}$, are large enough, we have

$$
\operatorname{card}\left(I_{k_{1}} \cap[n, m]\right)>N_{k_{1}}=\exp \left(K_{0}(\gamma \log 2)^{\beta} k_{1}^{\beta} 2^{(\gamma / \alpha)\left(k_{1}-1\right)}\right) \geq \exp \left(K_{0} 2^{k_{1}-1}\right)
$$

Hence, the arguments leading to (3.13) show that

$$
\frac{t_{m}}{t_{n}} \geq \exp \left(\exp \left(\frac{K_{0}}{4} 2^{k_{1}}\right)\right)
$$

The proof of Lemma 3.3 is now complete. 


\section{Proof of Theorem 1.3 : Part II}

Consider now the events $E_{n}=\left\{Y\left(t_{n}\right)<f\left(t_{n}\right)\right\}$. We have directly $\mathbb{P}\left(E_{n}\right)=b_{t_{n}}$, and $\sum_{n \in J} b_{t_{n}}=+\infty$, by Lemma 3.3. Therefore our set $J$ satisfies hypothesis (3.3). To verify (3.2), it suffices to prove the following statement

Given $\epsilon>0$, there exist a number $K$ and an integer $p$ such that

$$
\forall n \in J \quad n \geq p \Rightarrow \sum_{m \in J, m>n} \mathbb{P}\left(E_{n} \cap E_{m}\right) \leq \mathbb{P}\left(E_{n}\right)\left(K+(1+\epsilon) \sum_{m \in J, m>n} \mathbb{P}\left(E_{m}\right)\right) .
$$

Given $n \in J, J$ can be rewritten as follows $J=J^{\prime} \cup\left(\bigcup_{k \in \mathbb{N}} J_{k}\right) \cup J^{\prime \prime}$, where $J^{\prime}=\left\{m \in J, t_{n} \leq t_{m} \leq 2 t_{n}\right\}, J_{k}=$ $\left\{m \in J \cap I_{k}, t_{m}>2 t_{n}, \operatorname{card}\left(I_{k} \cap[n, m]\right) \leq \exp \left(K_{0} 2^{k-1}\right)\right\}$ and $J^{\prime \prime}=J-\left(J^{\prime} \cup\left(\bigcup_{k \in \mathbb{N}} J_{k}\right)\right)$.

Our first key small ball estimate is given in the following lemma.

Lemma 4.1. Consider $0<t<u$, and $\theta, \nu>0$. Then, we have

$$
\mathbb{P}\left(\left\{Y(t) \leq \theta t^{\gamma}\right\} \cap\{Y(u) \leq \nu\}\right) \leq \exp \left(K_{5}\right) \mathbb{P}\left(Y(t) \leq \theta t^{\gamma}\right) \exp \left(-\frac{K_{5}(u-t)}{\nu^{1 / \gamma}}\right)
$$

where $K_{5}$ depends on $H_{1}, H_{2}, H_{3}, \lambda_{1}$ and $\lambda_{2}$ only.

Proof. Set $F_{1}=\left\{Y(t) \leq \theta t^{\gamma}\right\}$ and $F_{2}=\{Y(u) \leq \nu\}$. Denote by $[x]$ the integer part of a real $x$. Let $\delta>0$. We consider the sequence $t_{k}, k \in\{0, . ., n\}$, where $t_{0}=t, t_{k+1}=t_{k}+\delta$ and $n=[(u-t) / \delta]$. We have

$$
\begin{aligned}
\mathbb{P}\left(F_{1}\right. & \left.\cap F_{2}\right)=\mathbb{P}\left(F_{1} \cap\left\{\sup _{0 \leq s \leq u} \sup _{0 \leq w_{1}, w_{2}, w_{3} \leq s}\left|X\left(w_{1}, w_{2}, w_{3}, s\right)\right| \leq \nu\right\}\right) \\
& \leq \mathbb{P}\left(F_{1} \cap\left\{\sup _{t \leq s \leq u} \sup _{0 \leq w_{1}, w_{2}, w_{3} \leq s}\left|X\left(w_{1}, w_{2}, w_{3}, s\right)\right| \leq \nu\right\}\right) \\
& \leq \mathbb{P}\left(F_{1} \cap\left\{\sup _{t \leq s \leq u} \sup _{0 \leq w_{1} \leq s}\left|\lambda_{1} s^{H_{2}+H_{3}} B_{H_{1}}\left(w_{1}\right)\right| \leq \nu\right\}\right) .
\end{aligned}
$$

Let $G_{k}$ be the event defined by

$$
G_{k}=F_{1} \cap\left\{\sup _{t \leq s \leq t_{k}} \sup _{0 \leq w_{1} \leq s}\left|\lambda_{1} s^{H_{2}+H_{3}} B_{H_{1}}\left(w_{1}\right)\right| \leq \nu\right\}
$$

We have $F_{1} \cap F_{2} \subset G_{k}$.

Moreover, we have

$$
G_{k+1} \subset G_{k} \cap\left\{\left|\lambda_{1} t_{k+1}^{H_{2}+H_{3}} B_{H_{1}}\left(t_{k+1}\right)-\lambda_{1} t_{k+1}^{H_{2}+H_{3}} B_{H_{1}}\left(t_{k}\right)\right| \leq 2 \nu\right\} .
$$

$X_{k}:=\lambda_{1} t_{k+1}^{H_{2}+H_{3}} B_{H_{1}}\left(t_{k+1}\right)-\lambda_{1} t_{k+1}^{H_{2}+H_{3}} B_{H_{1}}\left(t_{k}\right)$ can be rewritten by (1.1) as follows $X_{k}=X_{k, 1}+X_{k, 2}$, where

$$
X_{k, 1}=\lambda_{1} t_{k+1}^{H_{2}+H_{3}} k_{2 H_{1}}^{-1} \int_{t_{k}}^{t_{k+1}}\left(t_{k+1}-x\right)^{H_{1}-1 / 2} \tilde{W}(\mathrm{~d} x)
$$


Note also that $X_{k, 1}$ and $X_{k, 2}$ are independent.

Since $\mathbb{P}\left(\left|X_{k, 1}+x\right| \leq 2 \nu\right)$ is maximum at $x=0$ and $X_{k, 1}$ and $G_{k}$ are independent, we have

$$
\mathbb{P}\left(G_{k+1}\right) \leq \mathbb{P}\left(G_{k}\right) \mathbb{P}\left(\left|X_{k, 1}\right| \leq 2 \nu\right)
$$

The integral representation of $X_{k, 1}$ implies that $\mathbb{E}\left(X_{k, 1}\right)=0$ and

$$
\operatorname{Var} X_{k, 1}=\lambda_{1}^{2} t_{k+1}^{2\left(H_{2}+H_{3}\right)} k_{2 H_{1}}^{-2} \frac{\delta^{2 H_{1}}}{2 H_{1}} \geq \frac{\lambda_{1}^{2}}{2 H_{1} k_{2 H_{1}}^{2}} \delta^{2 \gamma}:=L^{2} \delta^{2 \gamma} .
$$

Denote by $\Phi$ the distribution function of the absolute value of a standard Gaussian random variable. Then, we obtain

$$
\mathbb{P}\left(G_{k+1}\right) \leq \mathbb{P}\left(G_{k}\right) \Phi\left(\frac{2 \nu}{L \delta^{\gamma}}\right)
$$

and therefore $\mathbb{P}\left(F_{1} \cap F_{2}\right) \leq \mathbb{P}\left(F_{1}\right) \Phi\left(\frac{2 \nu}{L \delta^{\gamma}}\right)^{n}$

Choosing $\delta=\nu^{1 / \gamma}$, we get $K_{5}=-\log \Phi\left(\frac{2}{L}\right)$. Lemma 4.1 is proved.

Lemma 4.2. $\sum_{m \in J^{\prime}} \mathbb{P}\left(E_{n} \cap E_{m}\right) \leq K^{\prime} b_{t_{n}}$ and $\sum_{m \in\left(\cup_{k} J_{k}\right)} \mathbb{P}\left(E_{n} \cap E_{m}\right) \leq K^{\prime \prime} b_{t_{n}}$, where $K^{\prime}$ and $K^{\prime \prime}$ are numbers.

Proof. Setting $u=t_{m}, t=t_{n}, \theta=a_{t_{n}}$ and $\nu=f\left(t_{m}\right)$, Lemma 4.1 implies

$$
\mathbb{P}\left(E_{n} \cap E_{m}\right) \leq \exp \left(K_{5}\right) b_{t_{n}} \exp \left(-\frac{K_{5}\left(t_{m}-t_{n}\right)}{f\left(t_{m}\right)^{1 / \gamma}}\right) .
$$

Consider first the case when $m \in J^{\prime}$.

Lemma 3.2 implies that, for all $i \geq n$, we have $t_{i+1}-t_{i} \geq t_{i} a_{t_{i}}^{1 / \gamma}=f\left(t_{i}\right)^{1 / \gamma} \geq f\left(t_{n}\right)^{1 / \gamma}$. Then we can establish

$$
t_{m}-t_{n} \geq(m-n) f\left(t_{n}\right)^{1 / \gamma} \text { and } f\left(t_{m}\right) \leq f\left(t_{n}\right)\left(\frac{t_{m}}{t_{n}}\right)^{\gamma} \leq 2^{\gamma} f\left(t_{n}\right) \text {. }
$$

Combining (4.2) with (4.3), we get

$$
\mathbb{P}\left(E_{n} \cap E_{m}\right) \leq \exp \left(K_{5}\right) b_{t_{n}} \exp \left(-\frac{K_{5}(m-n)}{2}\right)
$$

which is the first part of Lemma 4.2.

Consider now the case $m \in J_{k}$.

Combining (4.2) with the definition of $J_{k}$, we have

$$
\mathbb{P}\left(E_{n} \cap E_{m}\right) \leq \exp \left(K_{5}\right) b_{t_{n}} \exp \left(-\frac{K_{5}}{2\left(a_{t_{m}}\right)^{1 / \gamma}}\right) .
$$


Since $u(m)=k$, we get

$$
\mathbb{P}\left(E_{n} \cap E_{m}\right) \leq \exp \left(K_{5}\right) b_{t_{n}} \exp \left(-K_{5} 2^{k-1}\right),
$$

and consequently by noting that $\operatorname{card} J_{k} \leq \operatorname{card}\left(I_{k} \cap[n, m]\right) \leq \exp \left(K_{0} 2^{k-1}\right)$ and by assuming $K_{0}<K_{5}$, we have

$$
\sum_{m \in J_{k}} \mathbb{P}\left(E_{n} \cap E_{m}\right) \leq \exp \left(K_{5}\right) b_{t_{n}} \exp \left(\left(K_{0}-K_{5}\right) 2^{k-1}\right)
$$

Hence, Lemma 4.2 is proved.

To deal with the set $J^{\prime \prime}$, we recall the following three lemmas (see El-Nouty [9], p. 117, [11], p. 331, [11], p. 323).

Lemma A is a standard large deviation result for the sup-norm of a real-valued Gaussian process.

Lemma A. Let $\{Z(t), 0 \leq t \leq 1\}$ be a separable, centered, real-valued Gaussian process such that $Z(0)=0$, with probability 1 , and with incremental variance satisfying

$$
\left(\mathbb{E}(Z(t+h)-Z(t))^{2}\right)^{1 / 2} \leq f(h) \leq c_{f} h^{\beta_{1}}, \beta_{1}>0
$$

Then, we have for $c_{f}^{-1} \delta>1$

$$
\mathbb{P}\left(\sup _{0 \leq t \leq 1}|Z(t)| \geq \delta\right) \leq \frac{1}{C} \exp \left(-C\left(c_{f}^{-1} \delta\right)^{2}\right)
$$

where $C$ is a positive constant independent of $c_{f}$ and $\delta$.

Lemma B is the analogue of Lemma A for a two-parameter Gaussian process.

Lemma B. Let $X=\left\{X\left(s_{1}, s_{2}\right),\left(s_{1}, s_{2}\right) \in[0,1]^{2}\right\}$ be a separable real-valued centered Gaussian process such that $X(0,0)=0$ with Probability 1 and satisfying for any $\left[s_{1}, s_{1}+h_{1}\right] \times\left[s_{2}, s_{2}+h_{2}\right] \subset[0,1]^{2}$

$$
\left(\mathbb{E} X\left(\left[s_{1}, s_{1}+h_{1}\right] \times\left[s_{2}, s_{2}+h_{2}\right]\right)^{2}\right)^{1 / 2} \leq \kappa\left(h_{1}, h_{2}\right) \leq c_{\kappa} h_{1}^{\alpha_{1}} h_{2}^{\alpha_{2}}, \quad \alpha_{1}>0, \alpha_{2}>0
$$

where

and we write

$$
X\left(\left[s_{1}, t_{1}\right] \times\left[s_{2}, t_{2}\right]\right)=X\left(t_{1}, t_{2}\right)-X\left(s_{1}, t_{2}\right)-X\left(t_{1}, s_{2}\right)+X\left(s_{1}, s_{2}\right)
$$

$$
X\left(\left[s_{1}, t_{1}\right] \times\left[s_{2}, t_{2}\right]\right)=\int_{\left[s_{1}, t_{1}\right] \times\left[s_{2}, t_{2}\right]} X\left(\mathrm{~d}\left(u_{1}, u_{2}\right)\right) .
$$

Then, we have for $c_{\kappa}^{-1} \delta>1$

$$
\mathbb{P}\left(\sup _{\left(s_{1}, s_{2}\right) \in[0,1]^{2}}\left|X\left(s_{1}, s_{2}\right)\right| \geq \delta\right) \leq \frac{1}{C} \exp \left(-C\left(c_{\kappa}^{-1} \delta\right)^{2}\right)
$$

where $C$ is a positive constant independent of $c_{\kappa}$ and $\delta$. 
Lemma $\mathrm{C}$ derives from the existence of the right derivative of the convex function $\psi=-\log \phi$ and gives sharp bounds for the increments of $\phi$.

Lemma C. We have for $\epsilon_{1}>\epsilon / 2$ where $\epsilon$ is small enough

$$
\exp \left(-K_{3} \frac{\left|\epsilon_{1}-\epsilon\right|(\log (1 / \epsilon))^{\beta}}{\epsilon^{1+1 / \alpha}}\right) \leq \frac{\phi\left(\epsilon_{1}\right)}{\phi(\epsilon)} \leq \exp \left(K_{3} \frac{\left|\epsilon_{1}-\epsilon\right|(\log (1 / \epsilon))^{\beta}}{\epsilon^{1+1 / \alpha}}\right)
$$

where $K_{3}>0$.

Building on Lemmas A, B and C, we can establish our last key small ball estimate in the following result.

Lemma 4.3. Let $\lambda$ be a real number such that $1 / 2<\lambda<1$. Set

$$
r=\min \left(\frac{1-\max \left(H_{1}, H_{2}, H_{3}\right)}{3}, \frac{(1-\lambda) \alpha}{3}\right)
$$

Then, we have for $u \geq 2 t$

$$
\begin{aligned}
\mathbb{P}\left(Y(t) \leq \theta t^{\gamma}, Y(u) \leq \nu u^{\gamma}\right) \leq & \phi(\theta) \phi(\nu) \exp \left(2\left(\frac{t}{u}\right)^{r} K_{3}\left(\frac{\left(\log \frac{1}{\theta}\right)^{\beta}}{\theta^{1+(1 / \alpha)}}+\frac{\left(\log \frac{1}{\nu}\right)^{\beta}}{\nu^{1+(1 / \alpha)}}\right)\right) \\
& +3\left(\frac{1}{C_{1,2}} \exp \left(-\frac{C_{1,2}}{4 \lambda_{1}^{2} K_{H_{1}, 2}^{2}}\left(\frac{u}{t}\right)^{r}\right)+\frac{1}{C_{23,2}} \exp \left(-\frac{C_{23,2}}{4 \lambda_{2}^{2} K_{H_{2}, 2}^{2}}\left(\frac{u}{t}\right)^{r}\right)\right) \\
& +3\left(\frac{1}{C_{1,1}} \exp \left(-\frac{C_{1,1}}{4 \lambda_{1}^{2} K_{H_{1}, 1}^{2}}\left(\frac{u}{t}\right)^{r}\right)+\frac{1}{C_{23,1}} \exp \left(-\frac{C_{23,1}}{4 \lambda_{2}^{2} K_{H_{2}, 1}^{2}}\left(\frac{u}{t}\right)^{r}\right)\right),
\end{aligned}
$$

where $K_{H_{1}, 1}, K_{H_{1}, 2}>0$ depend on $H_{1}$ only, $K_{H_{2}, 1}, K_{H_{2}, 2}>0$ depend on $H_{2}\left(H_{2} \leq H_{3}\right)$ only, $K_{3}>0$ is defined as in Lemma $C, C_{1,1}, C_{1,2}>0$ are defined as in Lemma $A$ and $C_{23,1}, C_{23,2}>0$ are defined as in Lemma $B$.

Proof. Set $Q=\mathbb{P}\left(Y(t) \leq \theta t^{\gamma}, Y(u) \leq \nu u^{\gamma}\right)$.

Set $v=\sqrt{u t}$. If $t=o(u)$ then $t=o(v)$ and $v=o(u)$.

Using (1.1) and (1.2), we see that $B_{H_{1}}$ and $B_{H_{2}, H_{3}}$ can be split as follows

$$
B_{H_{1}}=B_{H_{1}, 1}+B_{H_{1}, 2} \text { and } B_{H_{2}, H_{3}}=B_{H_{2}, H_{3}, 1}+B_{H_{2}, H_{3}, 2},
$$

where

and

$$
B_{H_{1}, 1}\left(w_{1}\right)=\int_{\left|x_{1}\right| \leq v} g_{H_{1}}\left(w_{1}, x_{1}\right) \tilde{W}\left(\mathrm{~d} x_{1}\right),
$$

$$
B_{H_{2}, H_{3}, 1}\left(w_{2}, w_{3}\right)=\int_{\left|x_{2}\right| \leq v} \int_{-\infty}^{w_{3}} g_{H_{2}}\left(w_{2}, x_{2}\right) g_{H_{3}}\left(w_{3}, x_{3}\right) W\left(\mathrm{~d}\left(x_{2}, x_{3}\right)\right) .
$$

Note that $B_{H_{1}, 1}$ and $B_{H_{1}, 2}$ are independent as $B_{H_{2}, H_{3}, 1}$ and $B_{H_{2}, H_{3}, 2}$. 
(4.5) implies that the FMFBMFBS $X$ can be rewritten as follows $X=X_{1}+X_{2}$ where

$$
X_{1}\left(w_{1}, w_{2}, w_{3}, s\right)=\lambda_{1} s^{H_{2}+H_{3}} B_{H_{1}, 1}\left(w_{1}\right)+\lambda_{2} s^{H_{1}} B_{H_{2}, H_{3}, 1}\left(w_{2}, w_{3}\right) .
$$

Set

$$
Y_{i}(t)=\sup _{0 \leq s \leq t} \sup _{0 \leq w_{1}, w_{2}, w_{3} \leq s}\left|X_{i}\left(w_{1}, w_{2}, w_{3}, s\right)\right|, \quad t \geq 0, \quad i \in\{1,2\} .
$$

Then, given $\delta>0$, we have (Talagrand [24], pp. 210-211)

$$
Q \leq \phi(\theta+2 \delta) \phi(\nu+2 \delta)+3 \mathbb{P}\left(Y_{2}(t)>\delta t^{\gamma}\right)+3 \mathbb{P}\left(Y_{1}(u)>\delta u^{\gamma}\right)
$$

(4.4) implies

$$
\phi(\theta+2 \delta) \leq \phi(\theta) \exp \left(2 \delta K_{3}\left(\frac{\left(\log \frac{1}{\theta}\right)^{\beta}}{\theta^{1+(1 / \alpha)}}\right)\right)
$$

and consequently

$$
\phi(\theta+2 \delta) \phi(\nu+2 \delta) \leq \phi(\theta) \phi(\nu) \exp \left(2 \delta K_{3}\left(\frac{\left(\log \frac{1}{\theta}\right)^{\beta}}{\theta^{1+(1 / \alpha)}}+\frac{\left(\log \frac{1}{\nu}\right)^{\beta}}{\nu^{1+(1 / \alpha)}}\right)\right) .
$$

If we choose $\delta=\left(\frac{t}{u}\right)^{r}$, then we get the first term of the RHS of Lemma 4.3.

Next, we want to obtain an upper bound of

$$
\mathbb{P}\left(Y_{2}(t)>\delta t^{\gamma}\right)=\mathbb{P}\left(\sup _{0 \leq s \leq 1} \sup _{0 \leq w_{1}, w_{2}, w_{3} \leq s}\left|\lambda_{1} s^{H_{2}+H_{3}} L_{H_{1}, 2}\left(w_{1}\right)+\lambda_{2} s^{H_{1}} L_{H_{2}, H_{3}, 2}\left(w_{2}, w_{3}\right)\right|>\delta\right),
$$

where

$$
L_{H_{1}, 2}\left(w_{1}\right)=\int_{\left|x_{1}\right| \geq v / t} g_{H_{1}}\left(w_{1}, x_{1}\right) \tilde{W}\left(\mathrm{~d} x_{1}\right)
$$

and

$$
L_{H_{2}, H_{3}, 2}\left(w_{2}, w_{3}\right)=\int_{\left|x_{2}\right| \geq v / t} \int_{-\infty}^{w_{3}} g_{H_{2}}\left(w_{2}, x_{2}\right) g_{H_{3}}\left(w_{3}, x_{3}\right) W\left(\mathrm{~d}\left(x_{2}, x_{3}\right)\right) .
$$

We can show, by standard computations, that

$$
\mathbb{P}\left(Y_{2}(t)>\delta t^{\gamma}\right) \leq \mathbb{P}\left(\sup _{0 \leq w_{1} \leq 1}\left|L_{H_{1}, 2}\left(w_{1}\right)\right|>\frac{\delta}{2\left|\lambda_{1}\right|}\right)+\mathbb{P}\left(\sup _{0 \leq w_{2}, w_{3} \leq 1}\left|L_{H_{2}, H_{3}, 2}\left(w_{2}, w_{3}\right)\right|>\frac{\delta}{2\left|\lambda_{2}\right|}\right) .
$$

There exists $K_{H_{1}, 2}>0$ such that

$$
\operatorname{Var}\left(L_{H_{1}, 2}\left(w_{1}+h_{1}\right)-L_{H_{1}, 2}\left(w_{1}\right)\right) \leq K_{H_{1}, 2}^{2}\left(\frac{v}{t}\right)^{2 H_{1}-2} h_{1}^{2} .
$$


Hence, we may apply Lemma A and we get

$$
\mathbb{P}\left(\sup _{0 \leq w_{1} \leq 1}\left|L_{H_{1}, 2}\left(w_{1}\right)\right|>\frac{\delta}{2\left|\lambda_{1}\right|}\right) \leq \frac{1}{C_{1,2}} \exp \left(-\frac{C_{1,2}}{K_{H_{1}, 2}^{2}(v / t)^{2 H_{1}-2}} \frac{\delta^{2}}{4 \lambda_{1}^{2}}\right) .
$$

Set $\sigma_{H_{2}, 2}$ the covariance function of the process $\left\{L_{H_{2}, 2}(s), 0 \leq s \leq 1\right\}$. Since

$$
\mathbb{E}\left(L_{H_{2}, H_{3}, 2}\left(w_{2}, w_{3}\right) L_{H_{2}, H_{3}, 2}\left(w_{2}^{\prime}, w_{3}^{\prime}\right)\right)=\sigma_{H_{2}, 2}\left(w_{2}, w_{2}^{\prime}\right) \times \sigma_{H_{3}}\left(w_{3}, w_{3}^{\prime}\right),
$$

we have for any $\left[s_{2}, s_{2}+h_{2}\right] \times\left[s_{3}, s_{3}+h_{3}\right] \subset[0,1]^{2}$

$$
\begin{aligned}
& \mathbb{E}\left(L_{H_{2}, H_{3}, 2}\left(\left[s_{2}, s_{2}+h_{2}\right] \times\left[s_{3}, s_{3}+h_{3}\right]\right)^{2}\right) \\
&= \mathbb{E}\left(\int_{\left[s_{2}, s_{2}+h_{2}\right] \times\left[s_{3}, s_{3}+h_{3}\right]} L_{H_{2}, H_{3}, 2}\left(\mathrm{~d}\left(w_{2}, w_{3}\right)\right) \times \int_{\left[s_{2}, s_{2}+h_{2}\right] \times\left[s_{3}, s_{3}+h_{3}\right]} L_{H_{2}, H_{3}, 2}\left(\mathrm{~d}\left(w_{2}^{\prime}, w_{3}^{\prime}\right)\right)\right) \\
& \leq \int_{s_{2}}^{s_{2}+h_{2}} \int_{s_{2}}^{s_{2}+h_{2}}\left|\sigma_{H_{2}, 2}\left(w_{2}, w_{2}^{\prime}\right)\right| \mathrm{d} w_{2} \mathrm{~d} w_{2}^{\prime} \times \int_{s_{3}}^{s_{3}+h_{3}} \int_{s_{3}}^{s_{3}+h_{3}}\left|\sigma_{H_{3}}\left(w_{3}, w_{3}^{\prime}\right)\right| \mathrm{d} w_{3} \mathrm{~d} w_{3}^{\prime} \\
&:=I \times I I .
\end{aligned}
$$

Consider II first. We get by the inequality of Cauchy-Schwarz

$$
I I \leq \int_{s_{3}}^{s_{3}+h_{3}} \int_{s_{3}}^{s_{3}+h_{3}} w_{3}^{H_{3}} w_{3}^{\prime} H_{3} \mathrm{~d} w_{3} \mathrm{~d} w_{3}^{\prime} \leq h_{3}^{2} .
$$

Let us turn to I.

A straight computation implies that there exists $K_{H_{2}, 2}>0$ depending on $H_{2}$ such that

$$
\mathbb{E}\left(L_{H_{2}, 2}\left(w_{2}\right)\right)^{2} \leq K_{H_{2}, 2}^{2} w_{2}^{2}(v / t)^{2 H_{2}-2},
$$

and consequently, by the inequality of Cauchy-Schwarz,

$$
\left|\sigma_{H_{2}, 2}\left(w_{2}, w_{2}^{\prime}\right)\right| \leq K_{H_{2}, 2}^{2} w_{2} w_{2}^{\prime}(v / t)^{2 H_{2}-2} .
$$

So we get

$$
I \leq K_{H_{2}, 2}^{2}(v / t)^{2 H_{2}-2} h_{2}^{2} .
$$

Hence, combining (4.8) with (4.9) and (4.10), we have

$$
\mathbb{E}\left(L_{H_{2}, H_{3}, 2}\left(\left[s_{2}, s_{2}+h_{2}\right] \times\left[s_{3}, s_{3}+h_{3}\right]\right)^{2}\right) \leq K_{H_{2}, 2}^{2}(v / t)^{2 H_{2}-2} h_{2}^{2} h_{3}^{2} .
$$


An application of Lemma B with $\alpha_{1}=\alpha_{2}=1, c_{\kappa}=K_{H_{2}, 2}\left(\frac{v}{t}\right)^{H_{2}-1}$ and $c_{\kappa}^{-1} \delta>1$, yields

$$
\mathbb{P}\left(\sup _{0 \leq w_{2}, w_{3} \leq 1}\left|L_{H_{2}, H_{3}, 2}\left(w_{2}, w_{3}\right)\right|>\frac{\delta}{2\left|\lambda_{2}\right|}\right) \leq \frac{1}{C_{23,2}} \exp \left(-\frac{C_{23,2}}{K_{H_{2}, 2}^{2}\left(\frac{v^{2}}{t^{2}}\right)^{H_{2}-1}} \frac{\delta^{2}}{4 \lambda_{2}^{2}}\right) .
$$

Set $\delta=\left(\frac{t}{u}\right)^{r}$. Recall that $v^{2}=u t$ and $r \leq \frac{1-\max \left(H 1, H_{2}, H_{3}\right)}{3}$. Combining (4.6) with (4.7) and (4.11), we get

$$
\mathbb{P}\left(Y_{2}(t)>\delta t^{\gamma}\right) \leq \frac{1}{C_{1,2}} \exp \left(-\frac{C_{1,2}}{4 \lambda_{1}^{2} K_{H_{1}, 2}^{2}}\left(\frac{u}{t}\right)^{r}\right)+\frac{1}{C_{23,2}} \exp \left(-\frac{C_{23,2}}{4 \lambda_{2}^{2} K_{H_{2}, 2}^{2}}\left(\frac{u}{t}\right)^{r}\right)
$$

which is the second term of the RHS of Lemma 4.3 .

Finally, we want to establish a similar result for $\mathbb{P}\left(Y_{1}(u)>\delta u^{\gamma}\right)$, which is smaller than

$$
\mathbb{P}\left(\sup _{0 \leq w_{1} \leq 1}\left|L_{H_{1}, 1}\left(w_{1}\right)\right|>\frac{\delta}{2\left|\lambda_{1}\right|}\right)+\mathbb{P}\left(\sup _{0 \leq w_{2}, w_{3} \leq 1}\left|L_{H_{2}, H_{3}, 1}\left(w_{2}, w_{3}\right)\right|>\frac{\delta}{2\left|\lambda_{2}\right|}\right)
$$

where

and

$$
L_{H_{1}, 1}\left(w_{1}\right)=\int_{\left|x_{1}\right| \leq v / u} g_{H_{1}}\left(w_{1}, x_{1}\right) \tilde{W}\left(\mathrm{~d} x_{1}\right)
$$

$$
L_{H_{2}, H_{3}, 1}\left(w_{2}, w_{3}\right)=\int_{\left|x_{2}\right| \leq v / u} \int_{-\infty}^{w_{3}} g_{H_{2}}\left(w_{2}, x_{2}\right) g_{H_{3}}\left(w_{3}, x_{3}\right) W\left(\mathrm{~d}\left(x_{2}, x_{3}\right)\right) .
$$

Since $1 / 2<\lambda<1$, there exists $K_{H_{1}, 1}>0$ such that

$$
\operatorname{Var}\left(L_{H_{1}, 1}\left(w_{1}+h_{1}\right)-L_{H_{1}, 1}\left(w_{1}\right)\right) \leq K_{H_{1}, 1}^{2}\left(\frac{v}{u}\right)^{2 H_{1}-2 \lambda H_{1}} h_{1}^{2 \lambda H_{1}}
$$

Hence, we may apply Lemma A and we get

$$
\mathbb{P}\left(\sup _{0 \leq w_{1} \leq 1}\left|L_{H_{1}, 1}\left(w_{1}\right)\right|>\frac{\delta}{2\left|\lambda_{1}\right|}\right) \leq \frac{1}{C_{1,1}} \exp \left(-\frac{C_{1,1}}{K_{H_{1}, 1}^{2}(v / u)^{2 H_{1}-2 \lambda H_{1}}} \frac{\delta^{2}}{4 \lambda_{1}^{2}}\right)
$$

Set $\sigma_{H_{2}, 1}$ the covariance function of the process $\left\{L_{H_{2}, 1}(s), 0 \leq s \leq 1\right\}$. Since

$$
\mathbb{E}\left(L_{H_{2}, H_{3}, 1}\left(w_{2}, w_{3}\right) L_{H_{2}, H_{3}, 1}\left(w_{2}^{\prime}, w_{3}^{\prime}\right)\right)=\sigma_{H_{2}, 1}\left(w_{2}, w_{2}^{\prime}\right) \times \sigma_{H_{3}}\left(w_{3}, w_{3}^{\prime}\right),
$$

we have for any $\left[s_{2}, s_{2}+h_{2}\right] \times\left[s_{3}, s_{3}+h_{3}\right] \subset[0,1]^{2}$

$$
\begin{aligned}
& \mathbb{E}\left(L_{H_{2}, H_{3}, 1}\left(\left[s_{2}, s_{2}+h_{2}\right] \times\left[s_{3}, s_{3}+h_{3}\right]\right)^{2}\right) \\
& \leq \int_{s_{2}}^{s_{2}+h_{2}} \int_{s_{2}}^{s_{2}+h_{2}}\left|\sigma_{H_{2}, 1}\left(w_{2}, w_{2}^{\prime}\right)\right| \mathrm{d} w_{2} \mathrm{~d} w_{2}^{\prime} \times \int_{s_{3}}^{s_{3}+h_{3}} \int_{s_{3}}^{s_{3}+h_{3}}\left|\sigma_{H_{3}}\left(w_{3}, w_{3}^{\prime}\right)\right| \mathrm{d} w_{3} \mathrm{~d} w_{3}^{\prime} .
\end{aligned}
$$


Then, there exists $K_{H_{2}, 1}>0$ such that

$$
\mathbb{E}\left(L_{H_{2}, H_{3}, 1}\left(\left[s_{2}, s_{2}+h_{2}\right] \times\left[s_{3}, s_{3}+h_{3}\right]\right)^{2}\right) \leq K_{H_{2}, 1}^{2}(v / u)^{2 H_{2}-2 \lambda H_{2}} h_{2}^{2} h_{3}^{2} .
$$

An application of Lemma B with $\alpha_{1}=\alpha_{2}=1, c_{\kappa}=K_{H_{2}, 1}\left(\frac{v}{u}\right)^{H_{2}-\lambda H_{2}}$ and $c_{\kappa}^{-1} \delta>1$, yields

$$
\mathbb{P}\left(\sup _{0 \leq w_{2}, w_{3} \leq 1}\left|L_{H_{2}, H_{3}, 1}\left(w_{2}, w_{3}\right)\right|>\frac{\delta}{2\left|\lambda_{2}\right|}\right) \leq \frac{1}{C_{23,1}} \exp \left(-\frac{C_{23,1}}{K_{H_{2}, 1}^{2}\left(\frac{v}{u}\right)^{2 H_{2}-2 \lambda H_{2}}} \frac{\delta^{2}}{4 \lambda_{2}^{2}}\right) .
$$

Set $\delta=\left(\frac{t}{u}\right)^{r}$. Recall that $v^{2}=u t$ and $r \leq \frac{(1-\lambda) \alpha}{3}$. Combining (4.12) with (4.13) and (4.14), we get

$$
\mathbb{P}\left(Y_{1}(u)>\delta u^{\gamma}\right) \leq \frac{1}{C_{1,1}} \exp \left(-\frac{C_{1,1}}{4 \lambda_{1}^{2} K_{H_{1}, 1}^{2}}\left(\frac{u}{t}\right)^{r}\right)+\frac{1}{C_{23,1}} \exp \left(-\frac{C_{23,1}}{4 \lambda_{2}^{2} K_{H_{2}, 1}^{1}}\left(\frac{u}{t}\right)^{r}\right)
$$

which concludes the proof of Lemma 4.3.

Finally, we state the last technical lemma.

Lemma 4.4. There exists an integer $p$ such that, if $n>\sup _{s \leq p}\left(\sup I_{s}\right)$, then, for $m \in J$ ", $m>n$, given $\epsilon>0$, we have $\mathbb{P}\left(E_{n} \cap E_{m}\right) \leq(1+\epsilon) b_{t_{n}} b_{t_{m}}$.

Proof. Let $u(n)=k^{\prime}$ and $u(m)=k_{1}$. We have by Lemma 3.3

$$
\frac{t_{m}}{t_{n}} \geq \exp \left(\exp \left(\frac{K_{0}}{4} 2^{\min \left(k^{\prime}, k_{1}\right)}\right)\right)
$$

Let $p \in \mathbb{N}$. Then $k^{\prime}>p$ and $k_{1}>p$. Thus, we have $\min \left(k^{\prime}, k_{1}\right)>p$.

Set $t=t_{n}, u=t_{m}, \theta=a_{t_{n}}$ and $\nu=a_{t_{m}}$. Note that $\log \frac{1}{\theta} \leq \frac{1}{\theta} \leq 2^{\left(k^{\prime}+1\right) \alpha}, \quad \log \frac{1}{\nu} \leq \frac{1}{\nu} \leq 2^{\left(k_{1}+1\right) \alpha}$ and $\frac{1}{b_{t_{n}} b_{t_{m}}}=\exp (\psi(\theta)+\psi(\nu))$.

By using Lemma 4.3 and letting $p \rightarrow+\infty$, we complete the proof of Lemma 4.4.

Combining Lemma 4.2 and Lemma 4.4, we get (4.1). Since our set $J$ satisfies hypothesis (3.2) and (3.3), (3.4) implies that, given $\epsilon>0$,

$$
\frac{1}{1+2 \epsilon} \leq \mathbb{P}\left(\bigcup_{n \in J} E_{n}\right)=\mathbb{P}\left(\bigcup_{n \in J}\left\{Y\left(t_{n}\right) \leq f\left(t_{n}\right)\right\}\right)
$$

and consequently $f \in L U C(Y)$. The proof of Theorem 1.3 is now complete.

Acknowledgements. The author expresses his warmest thanks to the anonymous referees for pointing out reference [16] and numerous suggestions. 


\section{REFERENCES}

[1] A. Ayache, S. Leger and M. Pontier, Drap Brownien fractionnaire. Potential Anal. 178 (2002) 31-43.

[2] A. Ayache and Y. Xiao, Asymptotic properties and Hausdorff dimensions of fractional Brownian sheets. J. Fourier Anal. Appl. 11 (2005) 407-439.

[3] E. Belinsky and W. Linde, Small Ball Probabilities of Fractional Brownian Sheets via Fractional Integration Operators. J. Theoret. Probab. 15 (2002) 589-612.

[4] C. Borell, Convex measures on locally convex space. Math. Ark. Math. 12 (1974) 239-252.

[5] P. Cheridito, Mixed fractional Brownian motion. Bernoulli 7 (2001) 913-934.

[6] N.J. Cutland, P.E. Kopp and W. Willinger, Stock price returns and the Joseph effect: a fractional version of the Black-Scholes model. in Seminar on Stochastic Analysis, Random Fields and Applications, Progr. Probab. E. Bolthausen, M. Dozziand F. Russo Eds., Basel: Birkhauser 36 (1995) 327-351.

[7] C. El-Nouty, On the lower classes of fractional Brownian motion. Studia Sci. Math. Hungar. 37 (2001) 363-390.

[8] C. El-Nouty, Lower classes of fractional Brownian motion under Hölder norms, Limit Theorems in Probability and Statistics, Balatonlelle, 1999, I. Berkes, E. Csáki, M. Csörgő Eds., János Bolyai Mathematical Society, Budapest (2002) 7-34.

[9] C. El-Nouty, The fractional mixed fractional Brownian motion. Statist. Probab. Lett. 65 (2003) 111-120.

[10] C. El-Nouty, Lower classes of integrated fractional Brownian motion. Studia Sci. Math. Hungar. 41 (2004) 17-38.

[11] C. El-Nouty, The influence of a log-type small ball factor in the study of the lower classes. Bull. Sci. math. 129 (2005) 318-338.

[12] F. Gassmann and D. Bürki, Experimental investigation of atmospheric dispersion over the Swiss Plain - Experiment SIESTA, Boundary-Layer Meteorology, Springer Netherlands 41 (1987) 295-307.

[13] F. Gassmann, P. Gaglione, S.E. Gryning, H. Hasenjäger, E. Lyck, H. Richner, B. Neiniger, S. Vogt and P. Thomas, Experimental Investigation of Atmospheric Dispersion over Complex Terrain in a Prealpine Region (experiment SIESTA) Swiss Federal Institute for Reactor Research EIR 604 (1986).

[14] T. Kühn and W. Linde, Optimal series representation of fractional Brownian sheets. Bernoulli 8 (2002) 669-696.

[15] M. Ledoux and M. Talagrand, Probability in Banach spaces. Springer Verlag, Berlin (1994).

[16] W.V. Li, A Gaussian correlation inequality and its applications to small ball probabilities. Elect. Comm. in Probab. 4 (1999) $111-118$.

[17] W.V. Li and W. Linde, Existence of small ball constants for fractional Brownian motions. C. R. Acad. Sci. Paris 326 (1998) 1329-1334

[18] W.V. Li and Q.M. Shao, Gaussian Processes: Inequalities, Small Ball Probabilities and Applications, Stochastic Processes: Theory and Methods, Handbook of Statistics 19 (2001).

[19] M.A. Lifshits, Gaussian Random Functions. Kluwer Academic Publishers, Dordrecht (1995).

[20] D.M. Mason and Z. Shi, Small Deviations for Some Multi-Parameter Gaussian Processes. J. Theoret. Probab. 14 (2001) 213-239.

[21] D. Monrad and H. Rootzen, Small values of Gaussian processes and functional laws of the iterated logarithm. Probab. Theory Related Fields 101 (1995) 173-192.

[22] P. Révész, Random walk in random and non-random environments, World Scientific Publishing Co., Teaneck, NJ (1990).

[23] P.A. Samuelson, Rational theory of warrant pricing. Indust. Management Rev. 6 (1965) 13-31.

[24] M. Talagrand, Lower classes of fractional Brownian motion. J. Theoret. Probab. 9 (1996) 191-213.

[25] Y. Xiao and T. Zhang, Local times of fractional Brownian sheets. Probab. Theory Related Fields 124 (2002) 204-226. 